\title{
Algorithm Optical Codes: An Alternative to Random Optical Codes in an Intra-Satellite Optical Wireless Network
}

\author{
Jesus A. Martin-Gonzalez, Enrique Poves, Francisco J. Lopez-Hernandez \\ Dept. of Photonics Technology, Universidad Politecnica de Madrid \\ ETSIT, Av. Complutense 28040 Madrid, Spain \\ emails: jesus.martin@tfo.upm.es,kike@tfo.upm.esanddxtn@tfo.upm.es
}

\begin{abstract}
We propose a novel family of optical codes to be used in intra-satellite optical wireless networks. They are Algorithm Optical Codes (AOCs). This family is related with the recently introduced family Random Optical Codes (ROCs). Both families of codes are designed to be used in optical Code-Division Multiple-Access (OCDMA) systems. Similarly than their predecessor, i.e. ROCs, AOCs are specially suitable for optical networks where the number of channels varies, ever large, low bit rate requirements, energy limitations and packed data are presented. For example sensor networks demand these requirements.

A detailed description of AOCs is shown in this paper. Furthermore a comparison between them and ROCs is presented. We find that AOCs provide less probability of error than ROCs, for a given length of the code and a number of users. Moreover a system using AOCs can change the length of the code easier than a system using ROCs. This fact permits a more efficient accommodation to the actual number of users.

Finally the implementation in an intra-spacecraft Telecommand and Telemeasurement (TC/TM) optical wireless network is also described. We compare the families in the intra-spacecraft optical wireless network. Keywords: intra-satellite optical wireless networks, algorithm optical codes (ROCs), random optical codes (ROCs), optical codes, generalized optical orthogonal codes (OOCs), optical code-division multipleaccess (OCDMA).
\end{abstract}

\section{INTRODUCTION}

In this paper we propose an alternative to the Random Optical Codes (ROCs) for intra-satellite optical wireless networks introduced by the authors in [1], [2]. The proposed family is Algorithm Optical Codes (AOCs).

Both families are used in Optical Code-Division Multiple-Access (OCDMA) systems. In particular we focus on networks of sensors. The large number of users, simplicity of the implementation, number of channel adaptation and low duty cycle is a must in these applications.

Incoherent, intensity encoding/decoding techniques have been commonly implemented in Optical CodeDivision Multiple-Access (OCDMA) systems. Signature sequences as optical orthogonal codes (OOCs) [3]-[6], prime sequences [7]-[9] or $2^{n}$ prime codes [10]-[12] have been used in OCDMA systems. Recently, Salehi has revised many of these codes used in OCDMA systems in [13]. There, it is highlighted how the multiple-access interference (MAI) effect reduction has been deeply studied in the literature [14]-[18]. However an important step in OOCs evolution was the analysis of the performance for Generalized OOCs [19]. OOCs with any correlation value were considered. Furthermore the optimal parameters were found based on the exact probability of error.

Following the unrestricted correlation idea, ROCs [1], [20] present also several important features used in some OCDMA systems as sensor networks. Furthermore, the proposed family, i.e. AOCs, provide additional improvements keeping the advantages of ROCs. The most important is the dynamical accommodation of the length of the code.

The system model using AOCs is rigorously described in Sec. 2. The procedures to optimize the model are described in Sec. 3. The comparison between systems using respectively ROCs and AOCs is presented in Sec. 4. An example of a system where AOCs are used is shown in Sec. 5.

\section{SYSTEM MODEL}

The model is an on-off keying (OOK) OCDMA system. The phase synchronism between the emitter and the intended receiver is assumed. However others channels could be randomly delayed. Perfect chip synchronization is assumed, i.e. frequency locking. Thus, the channels are asynchronous incoherent and additives. The number of users is $N+1$.

An algorithm builds the codes as follows. Let $\left\{\xi_{i}^{(k)}\right\}_{i=1}^{\infty}$ be an infinite pseudo-random sequence of Bernoulli random variables (r.v.), for each channel. It is identically repeated by encoder and decoder starting in a common seed.

The work presented in this paper was supported by the European Space Agency (ESA) project: "Optical wireless IntraSpacecraft Communication" ESA Contract 19545/06/NL/GLC. 


$$
\xi_{i}^{(k)}=\left\{\begin{array}{c}
\text { Yes } \\
\text { No }
\end{array} i \in \mathbb{N}, k=\{1, \ldots, N+1\} .\right.
$$

Let $p$ defined by the probability of a success. Hence, the probability mass function (PMF) of $\left\{\xi_{i}^{(k)}\right\}_{i=1}^{\infty}$ is written as,

$$
P M F=\left\{\begin{array}{l}
\mathrm{P}\left(\xi_{\mathrm{i}}^{(\mathrm{k})}=\mathrm{Yes}\right)=p \\
\mathrm{P}\left(\xi_{\mathrm{i}}^{(\mathrm{k})}=\mathrm{No}\right)=1-p
\end{array}\right.
$$

We denote by marked the chip $i$, if $\xi_{i}^{(k)}=$ Yes. Thus, $p$ is the frequency of these marked chips.

In order to track the chips where the marked chips are, the information is stored in numerical variables $\left\{\theta_{n}^{(k)}\right\}_{n=1}^{\infty}$. Any $\theta_{n}^{(k)}$ is defined as the $n^{\text {th }}$ marked chip for the user $(k)$, i.e.

$$
\begin{aligned}
& \theta_{n}^{(k)}=j, n=1, \text { when } \xi_{1}^{(k)}=\text { No } \cap \ldots \cap \xi_{j-1}^{(k)}=\text { No } \cap \xi_{j}^{(k)}=\text { Yes }, \\
& \theta_{n}^{(k)}=j, n=\{2, \ldots, \infty\}, \text { when } \xi_{\theta_{(n-1)}^{(k)}}^{(k)}=\text { No } \cap \ldots \cap \xi_{j-1}^{(k)}=\text { No } \cap \xi_{j}^{(k)}=\text { Yes . }
\end{aligned}
$$

The weight $(w)$ of the code is the number of marked chips used to transmit a bit. The length $(L)$ is the number of chips that the sequence has to wait before to obtain $w$ marked chips. Furthermore, $L$ is the position of the $w^{\text {th }}$ marked chip, i.e. $L=\theta_{w}$. Notice that each bit could use sequences with different values of $L$. Moreover $L$ is a random variable. It measures the number of trials until the $w^{\text {th }}$ success of a Bernoulli random variable. Thus, $L$ have Negative Binomial distribution with parameters $w$ and $p$, thus $E[L]=\frac{w}{p}$, where $E[L]$ is the expectation of $L$. This fact allows us to give the expectation of the bit rate of the model.

The model is easily interpreted if we consider the expectation of $L$ as a parameter of the system. Notice that $p$ can be tailored in order to get the desirable $E[L]$ for our system, i.e. $p=\frac{w}{E[L]}$.

A scheme of the model is shown in Fig. 1.

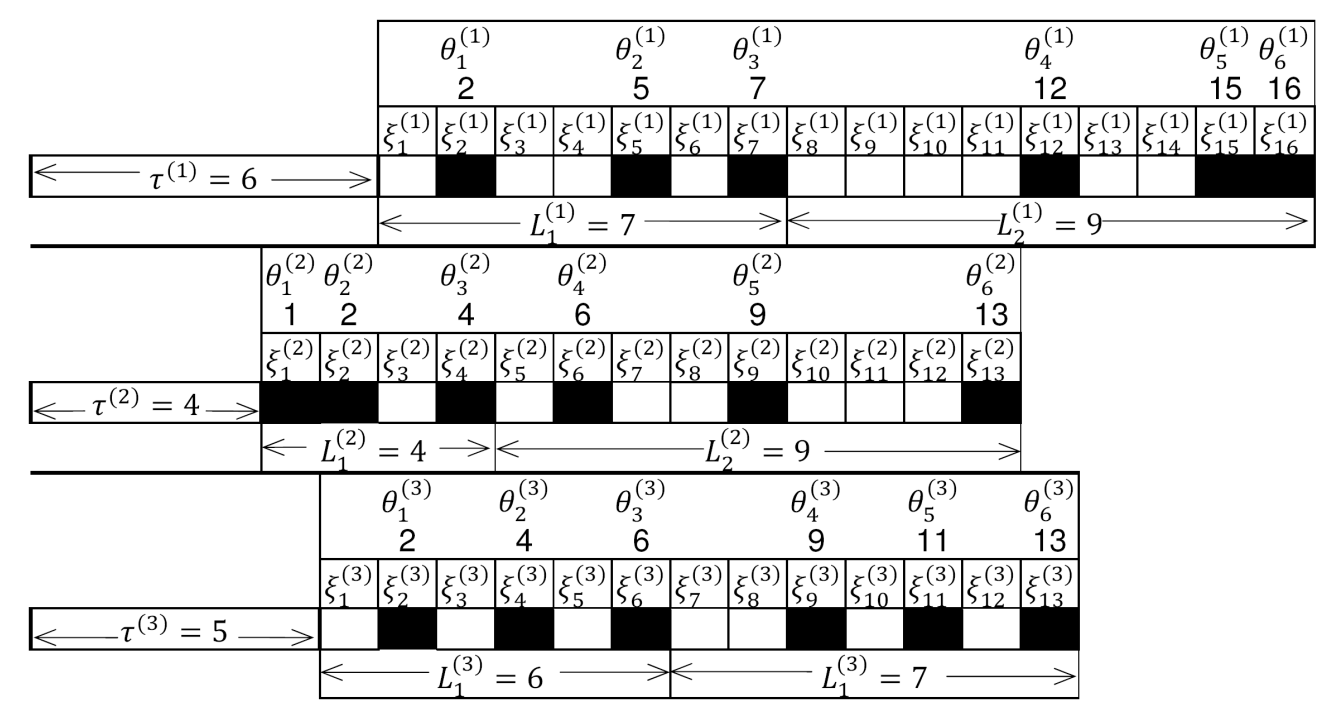

Fig. 1. Scheme of the model.

\section{OPTIMIZATION}

In this section the parameters of the model are optimized for systems using ROCs and AOCs. In both cases the parameters of the model are the length of the code $(L)$, the weight $(w)$ and the number of users in the model $(N+1)$.

The performance of the system is described by the following expressions for the probability of error $(P e)$. It is a function of the parameters aforementioned.

- Probability of error for ROCs $\left(P_{e}^{R O C s}\right)$, by [2]: 


$$
P_{e}^{R O C S}=\frac{1}{2^{N+1}} \sum_{i=0}^{N}\left(\begin{array}{c}
N \\
i
\end{array}\right)\left(1-\left(1-\frac{w}{L}\right)^{i}\right)^{w},
$$

- Probability of error for AOCs $\left(P_{e}^{A O C s}\right)$, by [21]:

$$
P_{e}^{A O C s}=\frac{1}{2}\left(1-\left(1-\frac{w}{2 E[L]}\right)^{N}\right)^{w} .
$$

The optimization of the parameters in both cases is a numerical search of the lower $w$ reaching the minimum probability of error for a given $L$ and $N$.

\section{COMPARISON}

In this section the performance of two on-off keying (OOK) OCDMA systems with asynchronous incoherent additives channels are compared. One of them is widely described by the authors in [1] and [20]. The other one is analyzed in [21].

The comparison between the performance of these systems is based on the optimizations described in Sec. 3 . The optimizations is computed for codes shorter than 1000 and system supporting up to 100 users. Notice that $P e$ is an $L$ and $N$ function. This fact allows the plotting of the contour levels of $P e$ for ROCs (solid lines) and for AOCs (dashed lines) in Fig. 2.

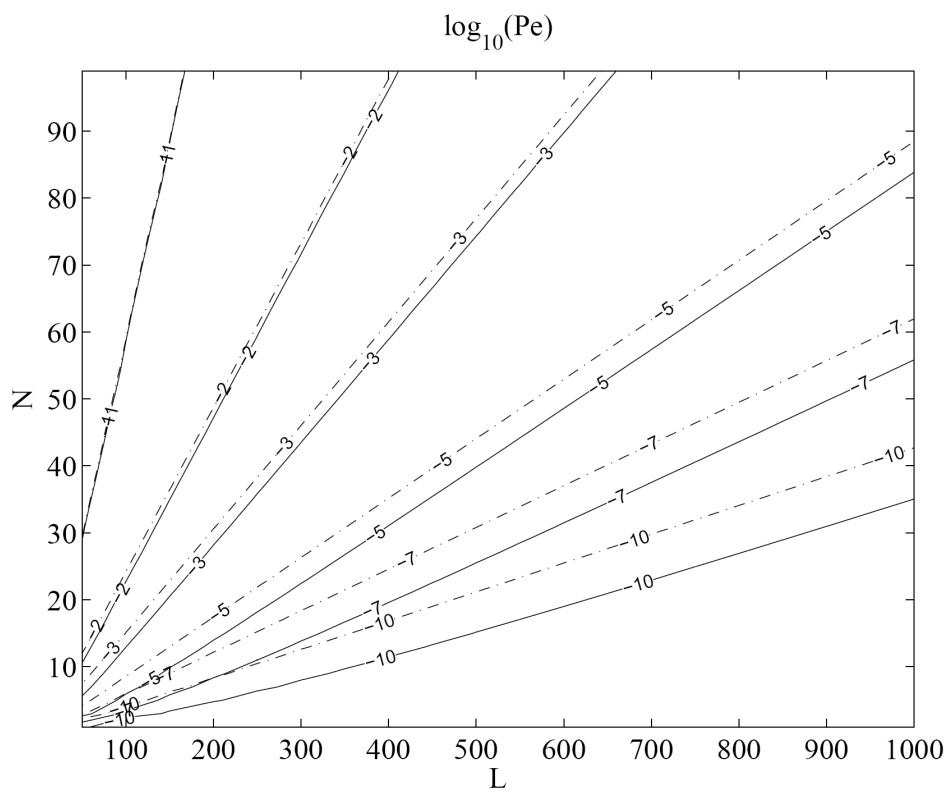

Fig. 2. Contour levels of $\log _{10}(P e)$. They are function of $L$ and $N$. Solid lines represent ROCs and dashes lines represent AOCs.

Results observed in Fig. 1 are the key to compare the performance of both families of codes. Comparison is shown for six different values of $P e$, i.e. $\left\{10^{-1}, 10^{-2}, 10^{-4}, 10^{-5}, 10^{-7}, 10^{-10}\right\}$. The best method is the one which allows a larger number of users for a given $L$ and $N$.

For a given $L$, AOCs accommodate more users than ROCs achieving the same value of probability of error. For example given $L=1000$, the number of users is 42 for AOCs and 35 for ROCs when $P e=10^{-10}$.

However the performance of the system is not the only advantage of AOCs versus ROCs. The dynamical accommodation to the actual number of users introduced by the authors in [2] using ROCs, can be easier implemented using AOCs. Notice that modifying only the parameter $p$, i.e. the frequency of the marked chips, the length of the code can be tuned. Based on this fact the system can be designed to adapt automatically $p$ to the actual number of users. This adaptation is identically predefined in the emitter and the received.

\section{APPLICATIONS AND EXAMPLES}

Based on the optimization found in Sec. 3, we can select the parameters for any application.

Following the same scenario introduced in [2], we design an intra-spacecraft Telecommand and Telemeasurement (TC/TM) optical wireless network using AOCs. 
In this system the On Board Computer (OBC) communicates with 25 terminals. The terminals include sensors and/or actuators thus the bit rate transmission could be low; actually $1 \mathrm{~kb} / \mathrm{s}$ per channel is the requirement. We use two channels per station (for upward and downward links), hence $N=49$.

Other constraining parameter is the probability of error. In this system it should be $P e \leq 10^{-7}$. In Fig. 2 $L=900$ is found for ROCs and $L=800$ for AOCs. Hence, it is shown than in this example, AOCs provide a shorter code then ROCs. In order to get the bit rate requested i.e. $1 \mathrm{~kb} / \mathrm{s}$, a chip period of $1.25 \mu$ is assigned.

\section{CONCLUSION}

We have shown the application of optical codes in an intra-satellite optical wireless network. In particular the use of a novel family of codes (AOCs) in this scenario is presented. The construction of this family is based in an algorithm and a common seed for the emitter and the receiver.

Moreover, the comparison between ROCs and AOCs used in OCDMA has been developed. AOCs present less probability of error than ROCs for any length and number of users.

We find AOCs as an alternative family of optical codes to be used in optical wireless networks. They keep the main features of ROCs: huge cardinality, adaptability to the system requirements, flexible duty cycle. Moreover they provide better performance an more capability of adaptation to the actual number of users.

\section{REFERENCES}

[1] J. A. Martin-Gonzalez, E. Poves, and F. J. Lopez-Hernandez, "Random optical codes in an intra-satellite optical wireless network." in IEEE ICTON Mediterranean Winter Conference, Dec. 2007.

[2] - "Random optical codes for optical code-division multiple-access." in IASTED International Conference on Wireless and Optical Communications (WOC 2008), May. 2008.

[3] J. A. Salehi, "Emerging optical code-division multiple-access communications systems," IEEE Netw., vol. 3, pp. 31-39, 1989.

[4] F. R. K. Chung, J. A. Salehi, and V. K. Wei, "Optical orthogonal codes: Design, analysis, and applications,” IEEE Trans. Inf. Theory, vol. 35, no. 3, pp. 595-604, May. 1989.

[5] J. A. Salehi, "Code division multiple-access techniques in optical fiber networks - Part I: Fundamental principles," IEEE Trans. Commun., vol. 37, no. 8, pp. 824-833, Aug. 1989.

[6] J. A. Salehi and C. A. Brackett, "Code division multiple-access techniques in optical fiber networks-Part II: Systems performance analysis," IEEE Trans. Commun., vol. 37, no. 8, pp. 834-842, Aug. 1989.

[7] A. A. Shaar and P. A. Davies, "Prime sequences - quasi-optimal sequences for or channel code division multiplexing," Electronics Letters, vol. 19, no. 21, pp. 888-890, 1983.

[8] P. R. Prucnal and M. A. Santoro, "Spread spectrum fiber optic local area network using optical-processing," Journal of Lightwave Technology, vol. 4, no. 5, pp. 547-554, May 1986.

[9] G. C. Yang and W. C. Kwong, "Performance analysis of optical CDMA with prime codes," Electronics Letters, vol. 31, no. 7, pp. 569-570, Mar. 301995.

[10] - "On the construction of 2 n codes for optical code-division multiple-access," IEEE Trans. Commun., vol. 43, no. 2-4, pp. 495-502, Feb.-Apr. 1995.

[11] W. C. Kwong and G. C. Yang, "Construction of 2 n prime-sequence codes for optical code division multiple access," IEE Proceedings- Communications, vol. 142, no. 3, pp. 141-150, Jun. 1995.

[12] W. Kwong, G. C. Yang, and J. Zhang, "2n prime-sequence codes and coding architecture for optical code-division multiple-access," IEEE Trans. Commun., vol. 44, no. 9, pp. 1152-1162, Sep. 1996.

[13] J. A. Salehi, "Emerging OCDMA communication systems and data networks [invited]," Journal of Optical Networking, vol. 6, no. 9, pp. 1138-1178, Sep. 12007.

[14] H. Chung and P. V. Kumar, "Optical orthogonal codes - new bounds and an optimal construction," IEEE Trans. Inf. Theory, vol. 36, no. 4, pp. 866-873, Jul. 1990.

[15] C. S. Weng and J. S. Wu, "Optical orthogonal codes with nonideal cross correlation," Journal of Lightwave Technology, vol. 19, no. 12, pp. 1856-1863, Dec. 2001.

[16] G. C. Yang and T. E. Fuja, "Optical orthogonal codes with unequal auto-correlation and cross-correlation constraints," IEEE Trans. Inf. Theory, vol. 41, no. 1, pp. 96-106, Jan. 1995.

[17] Y. Chang, R. Fuji-Hara, and Y. Miao, "Combinatorial constructions of optimal optical orthogonal codes with weight 4," IEEE Trans. Inf. Theory, vol. 49, no. 5, pp. 1283-1292, May 2003.

[18] Y. Chang and Y. Miao, "Constructions for optimal optical orthogonal codes," Discrete Mathematics, vol. 261, no. 1-3, pp. 127-139, Jan. 282003.

[19] S. Mashhadi and J. A. Salehi, "Code-division multiple-access techniques in optical fiber networks-Part III: Optical AND logic gate receiver structure with generalized optical orthogonal codes," IEEE Trans. Commun., vol. 54, no. 8, pp. 1457-1468, Aug. 2006.

[20] E. Poves, J. A. Martin-Gonzalez, and F. J. Lopez-Hernandez, "Use of optical orthogonal codes for intra-spacecraft communications." in IEEE ICTON Mediterranean Winter Conference, Dec. 2007.

[21] J. A. Martin-Gonzalez, E. Poves, and F. J. Lopez-Hernandez, "Comparison between the performance of algorithmic optical codes and orthogonal optical codes in OCDMA systems." in IEEE 10th International Symposium on Spread Spectrum Techniques and Applications. ISSSTA 2008, Aug. 2008, accepted for publication. 